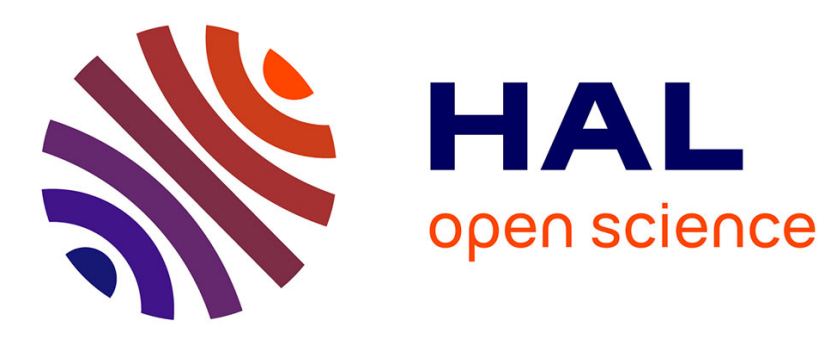

\title{
Belief scheduling for recognition of human action sequence
}

Emmanuel Ramasso, Denis Pellerin, Michèle Rombaut

\section{To cite this version:}

Emmanuel Ramasso, Denis Pellerin, Michèle Rombaut. Belief scheduling for recognition of human action sequence. 9th International Conference on Information Fusion, ICIF'06, IEEE/ISIF, 2006, Florence, Italy. hal-00068009

\section{HAL Id: hal-00068009 https://hal.science/hal-00068009}

Submitted on 10 May 2006

HAL is a multi-disciplinary open access archive for the deposit and dissemination of scientific research documents, whether they are published or not. The documents may come from teaching and research institutions in France or abroad, or from public or private research centers.
L'archive ouverte pluridisciplinaire HAL, est destinée au dépôt et à la diffusion de documents scientifiques de niveau recherche, publiés ou non, émanant des établissements d'enseignement et de recherche français ou étrangers, des laboratoires publics ou privés. 


\title{
Belief scheduling for recognition of human action sequence
}

\author{
E. Ramasso, D. Pellerin, M. Rombaut \\ Laboratoire des Images et des Signaux (LIS, UMR 5083) \\ 46, avenue Félix Viallet, 38031 Grenoble, France. \\ first_name.family_name@lis.inpg.fr
}

\begin{abstract}
In the context of human action sequence recognition in video sequences, a scheduler of actions is proposed. The Belief Scheduler is based on a Temporal Belief Filter ensuring a consistency in the temporal belief evolution as well as temporal constraints. The Belief Scheduler is inspired from System Science and is used to recognize actions in human activity defined as a sequence of actions. This new scheduler is proposed for applications in Computer Vision. The whole system of action and sequence recognition is based on the Transferable Belief Model proposed and developed by Ph. Smets which allows to explicitly model the doubt between actions. Furthermore, it emphasizes the conflict which is exploited for state change in action sequence. The experiments concern real video sequences with moving camera and several unknown view angles. The Temporal Belief Filter and the Belief Scheduler show their efficiency to cope with varying video quality and experiment conditions.
\end{abstract}

Keywords: human motion analysis, sequence analysis, constrained action recognition, scheduling, Transferable Belief Model.

\section{Introduction}

Human motion analysis is an important topic of interest in Computer Vision and Video Processing [1]. Research in these domains is motivated by the diversity of applications such as video indexing and retrieval, automatic surveillance and human-computer interaction. The main problem is to link the real world, which has intrinsically an analogical nature, to the human world which is symbolic [2].

Many methods have been proposed for human action sequence recognition [1] generally based on the Bayesian framework [3] with Hidden Markov Models (HMM) and Dynamic Bayesian Networks (DBN) [4].

In [5], an architecture was proposed for human action recognition using the Transferable Belief Model (TBM) [6]. The TBM is well-suited for action recognition notably because (i) doubtful transitions between actions are explicitly modelled, (ii) conflict between parameters reflects the need to improve the fusion process and (iii) reliability of parameters depends on the context and can be included in the system. In [7], a Temporal Belief Filter (TBF) was described for improving action recognition in the context of real video sequences with varying experiment conditions.

In this paper, a Belief Scheduler based on the TBM is proposed for human action sequence recognition. The Belief Scheduler exploits and embeds the TBF as well as scheduling principles [8]. Temporal sequences recognition is achieved by the scheduler by means of criteria based on conflict as defined in the TBM. In order to illustrate the proposed approach, athletics meeting videos acquired with a moving camera under an unknown view angle are analyzed to detect and recognize action sequences performed by one athlete.

The organization of the paper is as follows. An overview of the action recognition architecture based on the TBM is presented in Section 2. The main characteristics of the TBF are recalled in Section 3 to string with the Belief Scheduler described in Section 4. Section 5 deals with experimental results. Finally, we conclude and propose future work.

\section{Basic belief on actions ob- tained by fusion}

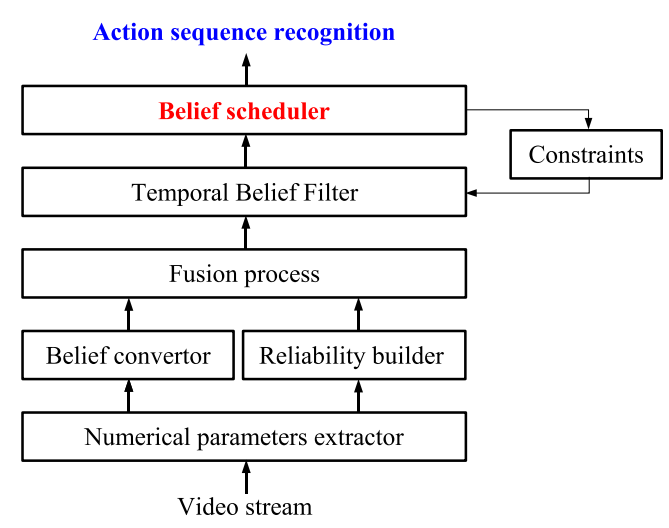

Figure 1: Architecture for human action sequence recognition in videos. The architecture is based on the Transferable Belief Model.

The whole system is presented in figure 1 . The video stream is analyzed by means of image processing. At each frame of the video, a belief is computed for each numerical parameter extracted from the video. These 
beliefs are then combined in the TBM framework. A detailed description of this part is given in [5] and the main points will be recalled in the sequel.

Some reliability factors are automatically determined at each frame of the video and inserted into the fusion process. The beliefs are then analyzed by the TBF described in [7] which provides smooth beliefs without conflict and with emphasized transitions. The TBF works on each action independently providing natural state of the actions. Causal relations and temporal constraints concerning actions are then added and managed in a scheduler of actions. As a result, belief on actions with constrained states are obtained. The Belief Scheduler is the main contribution of this paper.

\subsection{Numerical parameters}

Relevant numerical parameters are extracted at each frame from the video stream. Parameters are generally application dependant. In this paper, they are based on the following two main assumptions: first, the human is tracked by the cameraman because he is the center of interest and, second, the trajectories of human's head, center of gravity and one end of leg give information on actions.

The chosen parameters are (1) the camera motion parameters estimated from two successive frames and which are pan, tilt and zoom, and (2) the three human major points detected and tracked [5, 9]. Points coordinates are analytically combined to obtain more advanced parameters which are: the angle made by the human main axis and the horizon, the variation of the center of mass and the legs alternation.

\subsection{Transferable Belief Model fusion}

The numerical parameters values are converted into belief concerning the trueness of actions. A belief on actions is generated at each frame for each parameter. Belief of several parameters are then combined in the axiomatically well-founded Transferable Belief Model framework proposed by Smets and Kennes [6] to obtain a belief which takes all parameters into account.

\subsubsection{From numerical parameters to belief on actions}

An action $A$ is described by two hypotheses gathered in the frame of discernment (FoD) $\Omega_{A}=\left\{R_{A}, F_{A}\right\}$ with $R_{A}$ (resp. $F_{A}$ ) stands for "action $A$ is right" (resp. " $A$ is false"). In the sequel, an hypothesis concerning an action is called a state, e.g. "the current state of $A$ is $R_{A}{ }^{\prime}$.

In this part, the modelling is based on the assumption that actions are not exclusive, i.e. they can take place at the same time. They are also independant, i.e. the belief on one action does not imply anything about the belief of the others. Links between actions will be added by the Belief Scheduler described in Section 4.

The goal of the fusion process is to obtain the belief of each action $A$ according to several numerical parameters. A basic belief assignment (BBA) on an action $A$ according to a parameter $P$ is defined on the set of propositions $2^{\Omega_{A}}=\left\{\emptyset, R_{A}, F_{A}, R_{A} \cup F_{A}\right\}$ (where $R_{A} \cup F_{A}$ is the explicit doubt between hypotheses $R_{A}$ and $\left.F_{A}\right)$ by $m_{P}^{\Omega_{A}}: 2^{\Omega_{A}} \rightarrow[0,1], X \rightarrow m_{P}^{\Omega_{A}}(X)$ and by construction $m_{P}^{\Omega_{A}}(\emptyset)=0$, and $\sum_{X \subseteq \Omega_{A}} m_{P}^{\Omega_{A}}(X)=1$. A value $m_{P}^{\Omega_{A}}(X)$ is a basic belief mass which expresses a confidence in proposition $X \subseteq \Omega_{A}$ according to parameter $P$ but does not imply any additional claims regarding subsets of $X$. It is the fundamental difference with probability theory. A fuzzy-set inspired method is used to convert each numerical parameter into sources of belief.

\subsubsection{Fusion process}

Rules of combination are then applied to obtain a belief which takes the belief of all parameters into account. The fusion process is performed frame by frame for each action independently. Given two distinct BBAs $m_{P_{1}}^{\Omega_{A}}$ and $m_{P_{2}}^{\Omega_{A}}$ defined on the same FoD $\Omega_{A}$ then their combination is defined as:

$$
m_{P_{1}}^{\Omega_{A}} \otimes m_{P_{2}}^{\Omega_{A}}(E)=\sum_{C \triangle D=E} m_{P_{1}}^{\Omega_{A}}(C) \cdot m_{P_{2}}^{\Omega_{A}}(D)(1)
$$

with $\triangle=\cap$ (resp. $\cup$ ) for the conjunctive (resp. disjunctive) rule of combination. The rules of combination can be used in logical rules such as "if ... AND ... OR ...then ..." for describing actions by means of parameters states. These logical rules are then translated into belief combinations where the logical AND is replaced by the (-)-rule and the logical or by the (1)-rule assuming the same FoD [10].

\subsubsection{Integrating reliability}

The TBM offers a discounting process that allows to weight the belief of a parameter according to the reliability of the corresponding source. The reliability is an important tool for action recognition in video because it gives a penalty on belief provided by sources that work in non-optimal conditions. Given $\alpha_{P} \in[0,1]$ the reliability factor of a parameter $P$ then $1-\alpha_{P}$ is called the discount rate and the belief provided by the parameter is revised as:

$$
\begin{array}{lll}
m_{P}^{\Omega_{P}, \alpha_{P}}(A) & =\alpha_{P} \times m_{P}^{\Omega_{P}}, & \forall A \subsetneq \Omega_{P} \\
m_{P}^{\Omega_{P}, \alpha_{P}}\left(\Omega_{P}\right) & =\left(1-\alpha_{P}\right)+\alpha_{P} \times m_{P}^{\Omega_{P}} &
\end{array}
$$

Statistics can be used to compute the reliability factor [11]. In this work, reliability factors are automatically computed from data at each frame of the video and in an online manner to take into account the reliability that may vary, in particular according to the video quality. Two reliability factors are considered. The first one is based on the quantity of pixels belonging to the dominant motion in an image and gives an opinion concerning the quality of the camera motion estimation. The second one provides an opinion for the quality of the tracking algorithm and is computed as the normalized ratio between the distance "leg-center 
of gravity" and "head-center of gravity" which, physically, has to be close to 1 .

\section{$3 \quad$ Natural state estimation}

The Temporal Belief Filter (TBF) was proposed in a previous work described in [7]. The TBF works on each action independently taking as input the BBA obtained after parameters fusion and provides a BBA with the following properties:

- Consistency between parameters: the conjunctive rule of combination used in the TBM fusion process may emphasize a conflict between parameters which has to be solved,

- Temporal consistency: the belief on action can not vary abruptly between two successive frames,

- Exclusivity between action states: only one hypothesis concerning action (either $R_{A}$ or $F_{A}$ ) gets a no null belief at each frame.

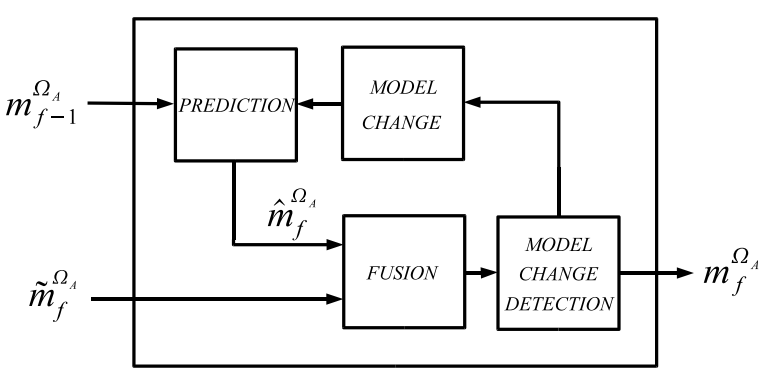

Figure 2: The Temporal Belief Filter (TBF) principle where $\hat{m}_{f}^{\Omega_{A}}$ is the prediction, $m_{f}^{\Omega_{A}}$ is the output value of the TBF at frame $f$ given the natural state of actions and $\tilde{m}_{f}^{\Omega_{A}}$ is the measure provided by the fusion of the parameters at frame $f$.

One important characteristic of the TBF is that it provides a BBA with only two focal sets: either $R_{A}$ and $R_{A} \cup F_{A}$, or $F_{A}$ and $R_{A} \cup F_{A}$. In the former case, the action is said to be in the right state while false state in the latter case. Both states are said to be natural because they are computed by the TBF for each action independently according to the BBA obtained after parameters fusion. These states will be called constrained if temporal constraints between actions are taken into account for the computation of an action state. In the sequel, the TBF is briefly described. Then, in Section 4, a method is proposed to add temporal constraints between actions.

The general principle of the TBF is depicted in Figure 2. The core of the TBF is based on implication rules which are well managed in the TBM framework. Details on their formalization as well as an application to target identification are described by Ristic and Smets in [10]. An implication rule is generally used to specialize a BBA. We have interpreted implication rules $\mathcal{R}$ and $\mathcal{F}$ as models of evolution denoted $\mathcal{M} \in\{\mathcal{R}, \mathcal{F}\}$. Each one focuses on one hypothesis of the FoD of an action $A$ which is either $R_{A}$ or $F_{A}$ states. At each frame $f$, the TBF works in two steps: (i) prediction, (ii) fusion and if necessary state change.

\subsection{Prediction}

The prediction step relies on the following assumption: if an action state is $R_{A}$ (resp. $\left.F_{A}\right)$ at frame $(f-1)$ then it would be partially $R_{A}$ (resp. $F_{A}$ ) at frame $f$. This model of evolution $\mathcal{R}$ (resp. $\mathcal{F}$ ) is weighted by a confidence value of $\gamma_{\mathcal{R}} \in[0,1]$ (resp. $\gamma_{\mathcal{F}} \in[0,1]$ ):

\section{Model $\mathcal{R}$ : \\ If $R_{A}$ at $(f-1)$ then $R_{A}$ at $f$ with belief of $\gamma_{R}$ \\ Model $\mathcal{F}$ : \\ If $F_{A}$ at $(f-1)$ then $F_{A}$ at $f$ with belief of $\gamma_{F}$}

In the sequel, the following vector notation of a BBA defined on a $\operatorname{FoD} \Omega_{A}$ is used:

$$
m^{\Omega_{A}}=\left[\begin{array}{llll}
m^{\Omega_{A}}(\emptyset) & m^{\Omega_{A}}\left(R_{A}\right) & m^{\Omega_{A}}\left(F_{A}\right) & m^{\Omega_{A}}\left(\Omega_{A}\right)
\end{array}\right]^{T}
$$

A model of evolution can be interpreted as a BBA. For instance, for the model $\mathcal{R}$ :

$$
m_{\mathcal{R}}^{\Omega_{A}}=\left[\begin{array}{llll}
0 & \gamma_{\mathcal{R}} & 0 & 1-\gamma_{\mathcal{R}}
\end{array}\right]^{T}
$$

The disjunctive rule of combination (Eq. 1) is then used to compute the prediction from the previous BBA and the model of evolution:

$$
\hat{m}_{f, \mathcal{M}}^{\Omega_{A}}=m_{\mathcal{M}}^{\Omega_{A}}(\mathrm{C}) m_{f-1}^{\Omega_{A}}
$$

The (U)-rule never assigns more belief to an hypothesis than does the previous BBA. As a clue, the prediction w.r.t. $\mathcal{R}$ (Eq. 6) is given by:

$$
\hat{m}_{f, \mathcal{R}}^{\Omega_{A}}=\left[\begin{array}{c}
0 \\
\gamma_{\mathcal{R}} \times m_{f-1}^{\Omega_{A}}\left(R_{A}\right) \\
0 \\
\left(1-\gamma_{\mathcal{R}}\right) \times m_{f-1}^{\Omega_{A}}\left(R_{A}\right)+m_{f-1}^{\Omega_{A}}\left(\Omega_{A}\right)
\end{array}\right]
$$

When $\gamma_{\mathcal{M}}=1$, the prediction equals the previous BBA reflecting a total confidence in the current state of action $A$. When $\gamma_{\mathcal{M}}=0$, the model expresses a total ignorance about the prediction of the current BBA on the action.

\subsection{Natural state change}

Prediction $\hat{m}_{f, \mathcal{M}}^{\Omega_{A}}$ and measure $\tilde{m}_{f}^{\Omega_{A}}$ represent two distinct pieces of information concerning the natural state of action $A$. The conjunctive combination (Eq. 1) of their associated BBA leads to a new BBA. If the sources are discordant, then a conflict is emphasized indicating a potential natural state change, i.e. the model might be changed. Thus, the conflict value $\epsilon_{f}$ (Eq. 7) seems relevant for model change requirement:

$$
\epsilon_{f}=\left(\hat{m}_{f, \mathcal{M}}^{\Omega_{A}} \bigcirc \tilde{m}_{f}^{\Omega_{A}}\right)(\emptyset)
$$

The conflict analysis is thus required to know whether the current model is no longer valid. The CUSUM process of the conflict is well adapted for solving 
problems concerning abrupt and short changes or gradual and long changes in the conflict value because it allows to sum up conflict during time. Generally, the CUSUM is the cumulative sum during time of the error between a prediction and a measure. In the case concerned, the error is the conflict value.

The initial Cusum process works as follows: when the CUSUM value becomes greater than a warning threshold $\mathcal{T}_{w}$ then the frame is stored as $f_{w}$ and the model is kept as valid. As soon as the CUSUM value becomes greater than a stop threshold $\mathcal{T}_{s}$ (at frame $f_{s}$ ) then the model is changed and the new model is applied from $f_{s}$. When a conflict appears between prediction and measure, as it could be the case in interval $\left[f_{w}, f_{s}\right]$, it was chosen to trust the model of evolution. Thus, the prediction is kept instead of an erroneous measurement and it avoids propagating conflict which is absorptive by the (-)-rule:

$$
m_{f}^{\Omega_{A}}= \begin{cases}\hat{m}_{f, \mathcal{M}}^{\Omega_{A}} @ \tilde{m}_{f}^{\Omega_{A}} & \text { if } \epsilon_{f}=0 \\ \hat{m}_{f, \mathcal{M}}^{\Omega_{A}} & \text { otherwise }\end{cases}
$$

Equation (8) accounts for the fact that the BBA $m_{f-1}^{\Omega_{A}}$ can have only two focal sets (Eq. 6) depending on the current model $\mathcal{M}$. Furthermore, the output of the TBF is a BBA without conflict and with only one hypothesis whose belief is not null. The interest of the (U)-rule is emphasized when there is often conflict because it allows to obtain $m_{f \rightarrow \infty}^{\Omega_{A}}\left(\Omega_{A}\right)=1$ which reflects total ignorance of the system.

To cope with low conflict during a long time, a fading memory process has been embedded which allows to forget gradually past event. The fading memory process requires a coefficient nicknamed fader, and denoted as $\lambda$, which works on the current CUSUM $\mathbf{C S}(f)$ as follows:

$$
\mathbf{C S}(f) \leftarrow \mathbf{C S}(f-1) \times \lambda+\epsilon_{f}
$$

The fader is here chosen as a constant and is applied at each frame.

The two models $(\mathcal{R}$ and $\mathcal{F}$ ) are tuned once and one model is applied while it is valid. Otherwise, it is changed by the other. A natural model change is required for an action $A_{k}$ when the stop threshold $\mathcal{T}_{w}^{k}$ is reached by its CUSUM. If the model change is accepted and performed, then the interval of frames $\mathbf{I}_{\mathbf{T}}=\left[f_{w}, \min \left(f_{s}, f_{w}+\mathcal{W}\right)\right]$ can be interpreted as an interval of transition between the two action states. The parameter $\mathcal{W}$ limits the size of the transition. The vacuous $\mathrm{BBA}$ is assigned to the frames belonging to $\mathbf{I}_{\mathbf{T}}$ to well represent ignorance: $m_{\mathbf{I}_{\mathbf{T}}}^{\Omega_{A}}\left(\Omega_{A}\right)=1$. After a model change, the new model is applied from the upper bound of the interval of transition $\mathbf{I}_{\mathbf{T}}$ and the CUSUM is reset.

Initialization - The $\mathrm{TBF}$ is an online process. In order to initialize the system, the model which better fits the first data is determined. For that, the CUSUM process is applied on an interval of frames for all models and the chosen one minimizes the CUSUM.

\subsection{Suggestions for parameters setting}

First, it is necessary to set the parameters in a relevant order: the fader and the models together, then the stop threshold, the warning threshold and at last the window. Second, for a given fader, the value of $\mathcal{T}_{s}$ can be estimated by $\hat{\mathcal{T}}_{s}$ if references concerning ground truth of actions are available i.e. the start frame $f_{\text {sref }}$ and the end frame $f_{\text {eref }}$ of these actions are known. For that, the filter has to be applied with a model of type $\mathcal{F}$ (false state) with a stop threshold $\mathcal{T}_{s}$ unreachable (close to infinity). Then, $\hat{\mathcal{T}}_{s}$ is estimated by $\hat{\mathcal{T}}_{s}=\mathbf{C S}\left(f_{\text {sref }}\right)$. If the data do not contain too much conflict, the estimation should be optimal otherwise, the fader has to be increased.

\section{Constrained state estimation}

In the previous section, action filtering was performed independently for each action. In this section, temporal constraints between actions are considered by assuming that the real state of an action is constrained by the activity defined as an ordered sequence of $K$ actions. The proposed algorithm for constrained state estimation relies on the TBF and is called Belief Scheduler.

\subsection{Sequence analysis problem}

In the context of temporal sequence analysis, the Directed Acyclic Graph (DAG) is one of the most common and used tool in Computer Vision for human action sequence recognition such as Dynamic Bayesian Networks [4]. Within the TBM framework, at least two solutions have been proposed in the literature. In [12], a belief Petri net was proposed but it is not enough robust for human action sequence recognition in case of noise data, with false alarms and delayed action. The Evidential Network (ENC) initially proposed by Smets and deeply studied by $\mathrm{Xu}$ and Smets in [13] represents another solution but conditional beliefs must be provided. ENCs are discussed and generalized in [14].

These tools have not been applied for human motion analysis yet. Recently, the Credal ExpectationMaximization algorithm (CrEM) [15] has been proposed and might be a powerful tool when coupled with a ENC.

The method proposed in this paper is inspired from the work of System Science community. More precisely, the main idea is based on scheduling as defined in the divisible load paradigm [8]. Scheduling is performed by an algorithm nicknamed scheduler which generally aims at executing tasks as fast as possible while respecting specifications given by the designer of the scheduler like the ordering of the tasks, their priority or the access control to resources. The scheduler 
is the core of an operating system such as Linux [16] and allows to optimize the programs execution while ensuring good performance for the user.

\subsection{Activity constraints}

An activity is defined as an ordered sequence of $K$ actions, $A_{1}, A_{2}, \ldots A_{k}, \ldots A_{K}$. Ideally, the actions are synchronized but in real videos, they can be either overlapped or unconnected such as represented in Figure 3. Three main reasons can be identified:

- it is physically impossible to determine crisp boundary between two consecutive actions,

- the thresholds setting are seldom perfect in the numeric-to-symbolic conversion or in the TBF.

- there is always noise on the numerical parameters leading to either overlapped or unconnected actions.

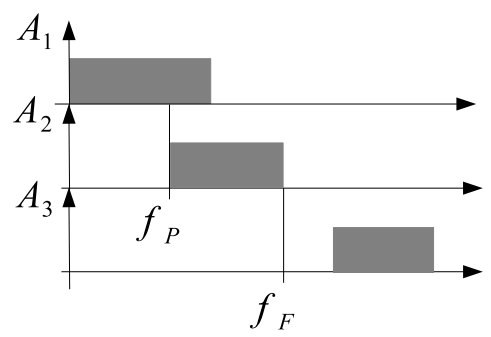

Figure 3: Representation of natural right state of actions using intervals (overlap between $A_{1}$ and $A_{2}$; gap between $A_{2}$ and $A_{3}$.

Overlapping and unconnection phenomenons are described as follows (where $f$ is the current frame):

- overlapped: at frame $f_{P}$ read as "the frame of preemption", two consecutive actions $A_{k}$ and $A_{k+1}$ have a natural right state at the same time.

- unconnected: at frame $f_{F}$ read as "the frame of forcing", an action $A_{k}$ becomes false while the next $A_{k+1}$ is not yet right. Thus, two actions have a natural false state at the same time.

As it has been seen in [7] and recalled Section 3, a model $\mathcal{R}$ or $\mathcal{F}$ is associated to each action according to its state. Thus, the models $\{\mathcal{R}, \mathcal{F}\}$ can be interpreted as resources while actions represent threads ${ }^{1}$ which try to access the resources. There are one resource of type $\{\mathcal{R}\}$ and $(K-1)$ resources of type $\{\mathcal{F}\}$. The access control is managed by the Belief Scheduler according to the information provided by the TBF concerning the model change process.

The Belief Scheduler relies on the following assumption: at each frame of the video, one and only one action is in the right state while the others are in the false state despite of the overlapping and gap problems. Therefore, the Belief Scheduler modifies the state of each action according to the state of its neighboring actions.

\footnotetext{
${ }^{1}$ Concept introduced in [3] for human motion analysis.
}

\subsection{Belief Scheduler description}

The Belief Scheduler has in charge to deal with the state change according to the data and the sequence. Its goal is to re-synchronize the actions by defining the constrained state for each of them. The proposed algorithm is based on the TBF and the diagram of the Belief Scheduler is depicted in Figure 4. One important characteristic of the Belief Scheduler is that it allows only one action to be true at a given time.

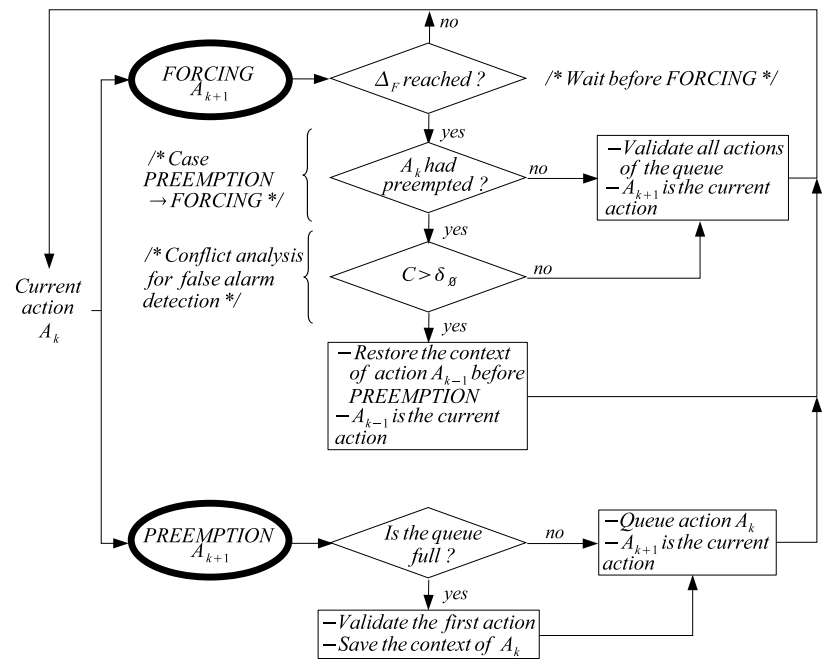

Figure 4: The Belief Scheduler of actions.

The algorithm considers two consecutive actions: the current one $A_{k}$ and its following $A_{k+1}$. Thus, the order in the sequence is taken into account. All actions are compelled to be in the false state with the model $\mathcal{F}$ except the current action $A_{k}$ which is in the right state with the model $\mathcal{R}$. If the CUSUM of $A_{k}$ or of $A_{k+1}$ exceeds their respective stop threshold, then the Belief Scheduler is informed that the sequence is in a transition step. The transition between two actions in the sequence is achieved by two processes: the PREEMPTION and the FORCING, for managing overlapped and unconnected actions respectively.

\subsubsection{Preemption process}
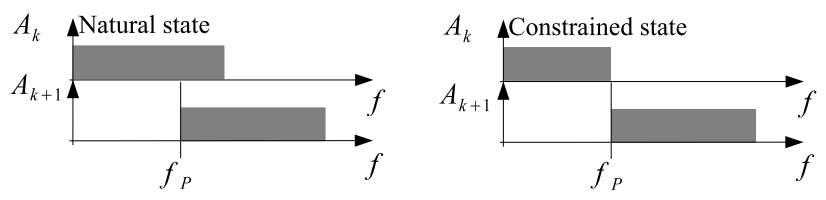

Figure 5: Preemption process.

This process concerns overlapped actions. At $\left(f_{P}-\right.$ 1 ), natural state of $A_{k}$ is right and natural state of $A_{k+1}$ is false. At $f_{P}, A_{k}$ is still right while $A_{k+1}$ becomes right thus, two actions are right at the same time. In this case, constrained state of $A_{k+1}$ is temporarily right (right state) from frame $f_{P}$ and the constrained state of $A_{k}$ is temporarily false (false state) until validation (see Figure 5 ). The validation is enabled when the quality of the recognition of the ac- 
tion which asks for preemption, e.g. $A_{k+1}$, is good (section 4.3.3). Information at $f_{P}$ concerning actions (cusum, belief,...), also called the context, is stored in case the PREEMPTION would not be enabled.

\subsubsection{Forcing process}

At $\left(f_{F}-1\right)$, the natural state of $A_{k}$ is right and the natural state of $A_{k+1}$ is false. At $f_{F}, A_{k}$ is false as well as $A_{k+1}$. Thus, the two actions are false at the same time. If the two successive actions are a little unconnected, i.e. with a small gap less than $\Delta_{F}$, constrained state of $A_{k}$ is forced to the right state until $A_{k+1}$ becomes right. However, sometimes, the gap between successive actions can be large, i.e. with a size greater than a value $\Delta_{F}$. The action requiring a FORCING, e.g. constrained state of $A_{k}$, keeps on being right until the frame $\left(f_{F}+\Delta_{F}\right)$. In this case, constrained state of $A_{k+1}$ is forced to be right and constrained state of $A_{k}$ becomes false (see Figure 6).
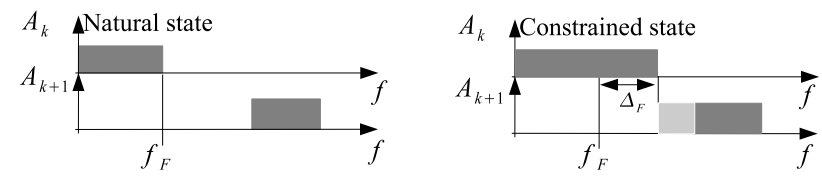

Figure 6: Forcing process.

In the case of FORCING, there is conflict between the natural and constrained states of actions $A_{k}$ and $A_{k+1}$. Therefore, according to Equations (6) and (8) embedded in the TBF, the belief tends to ignorance. If the gap is too large, i.e. more than $\Delta_{F}$, the constrained state of $A_{k+1}$ is always in conflict with its natural state. Thus, the TBF naturally changes it into the false state.

\subsubsection{False alarm detection}

A false alarm appears when an action becomes true instead of staying false. It corresponds to the case that an action $A_{k+1}$ tries to perform a FORCING on $A_{k+2}$ while, beforehand, $A_{k+1}$ had made a PREEMPTION on $A_{k}$. If actions $A_{k+1}$ and $A_{k+2}$ are too much unconnected, then $A_{k+1}$ can be interpreted as a false alarm (see Figure 7). This false alarm procedure is applied to validate a PREEMPTION.
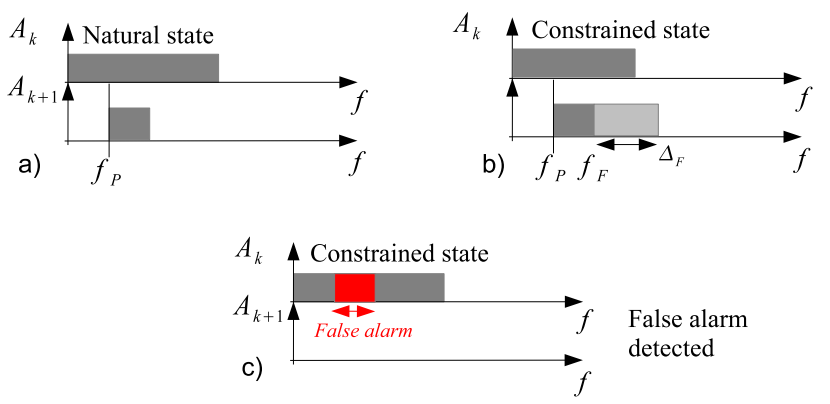

Figure 7: False alarm processing.

In order to decide whether action $A_{k+1}$ is or not a false alarm, it is required to assess the recognition performance of this action. Thus, there is a need to find a quality criterion in order to assess this performance. One difficulty is that the criterion has to be computed without references.

The chosen criterion is the mean of conflict, denoted $\bar{C}_{\emptyset}$, computed along the interval $\left[f_{P}, f_{F}+\Delta_{F}\right]$. We denote $N_{f}=\left(f_{F}+\Delta_{F}-f_{P}+1\right)$ the number of frames of the interval concerning the action $A_{k+1}$ which is potentially a false detection and which has made a PREEMPTION after a FORCING (Fig. 7,b). In this interval, the constrained state of the action $A_{k+1}$ is often in conflict with its natural state. The mean of conflict $\bar{C}_{\emptyset}$ is:

$$
\bar{C}_{\emptyset}=\frac{1}{N_{f}}(\underbrace{\sum_{f=f_{P}}^{f_{F}} \epsilon_{f}\left(A_{k+1}\right)}_{\text {natural conflict }}+\underbrace{\sum_{f=f_{F}+1}^{f_{F}+\Delta_{F}} \epsilon_{f}\left(A_{k+1}\right)}_{\text {artificial conflict }})
$$

where $\epsilon_{f}\left(A_{k+1}\right)$ is the conflict between measurement and model for action $A_{k+1}$ and given by Equation (7). If $\bar{C}_{\emptyset}>\delta_{\emptyset}$, where $\delta_{\emptyset}$ is a crisp threshold, the detection of the action is considered as a false detection. In consequence, the context of the actions at frame $f_{P}$, as for instance the value of the CUSUM, is restored and the previous action (before preemption), e.g. $A_{k}$, becomes right again. If $\bar{C}_{\emptyset}<\delta_{\emptyset}$ then $A_{k+2}$ becomes right and $A_{k+1}$ and $A_{k+2}$ are both validated.

\subsubsection{Elucidating the parameter $\Delta_{F}$}

To give a hand in the setting of the parameter $\Delta_{F}$, some details are given concerning its interpretation. Its value can be bounded if it is possible to estimate the higher value $D_{M}$ of the disjunction space between two states estimated or given by expert knowledge. In this case, $\Delta_{F} \geq D_{M} / 2$. In addition, the value of $\Delta_{F}$ should influence the value of $\bar{C}_{\emptyset}$ by increasing artificially the conflict. Given $\delta_{\emptyset}$, let $d_{m}$ be the minimal duration of an action estimated or given by expert knowledge. In this case, $\Delta_{F} /\left(\Delta_{F}+d_{m}\right) \leq \delta_{\emptyset}$. Therefore, if $D_{M}$ and $d_{m}$ are known then $\Delta_{F}$ can be bounded:

$$
\frac{D_{M}}{2} \leq \Delta_{F} \leq \frac{\delta_{\emptyset}}{1-\delta_{\emptyset}} \times d_{m}
$$

Learning $D_{M}$ and $d_{m}$ is quite straightforward if references are given. Concerning $\delta_{\emptyset}$, its setting depends on the setting of the TBF. Its value was set empirically to 0.2 for all tests.

\subsubsection{Queuing}

When several actions perform consecutive PREEMPTION, they must be validated to ensure they are not false alarms. One solution consists in storing them in a FIFO queue to wait for their validation. As emphasized in the previous section, the validation will appear after a FORCING process. The number of actions in the queue is limited and when the queue is full, the first queued-action is automatically validated.

Initialization - It can be noticed that, at the beginning of the scheduling, all actions are in the false state. An artificial initial right state action is added in 
the sequence that allows the Belief Scheduler to wait for a PREEMPTION of the first action.

\section{Experiments}

Database description: The proposed system is used to recognize high jump and pole vault actions sequence. Their sequence are running, jumping and falling.
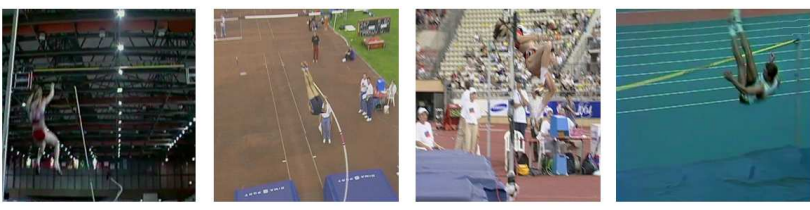

Figure 8: Heterogeneous database used for testing.

The database is composed of 40 videos acquired with a moving camera and several unknonw view angles. There are 25 pole vaults and 15 high jumps equivalent to about 6500 frames. The database is characterized by its heterogeneity (Fig. 8) with a panel of view angles as well as environments and athletes (out/indoor, male, female, other moving people).

Settings: To show the generalization capabiblity of the method, the TBF parameters as well as the the Belief Scheduler ones are the same for all actions and all videos. The parameters are tuned using the elucidations provided in the previous sections (§ 3.3 and 4.3.4) and expert knowledge. For information we set: $\lambda=0.9, \gamma_{\mathcal{R}}=\gamma_{\mathcal{F}}=0.9, \mathcal{T}_{s}=3, \mathcal{T}_{w}=0.5$, $\mathcal{W}=5, \delta_{\emptyset}=0.2$ and $\Delta_{F}=8$.

Decision-taking based on belief: We use the pignistic probability (BetP) proposed by $\mathrm{Ph}$. Smets [17]. We focus on the BetP of actions which are true (right state) therefore:

$$
\operatorname{BetP}\left(\mathrm{R}_{\mathrm{A}}\right)=\frac{1}{(1-\mathrm{m}(\emptyset))}\left(\mathrm{m}\left(\mathrm{R}_{\mathrm{A}}\right)+\frac{\mathrm{m}\left(\mathrm{R}_{\mathrm{A}} \cup \mathrm{F}_{\mathrm{A}}\right)}{2}\right)
$$

If $\operatorname{BetP}\left(\mathrm{R}_{\mathrm{A}}\right)>0$ then $A$ is considered as true.

Evaluation criteria: The database is annotated. We assess, first, the actions within the sequences and then, the beginning and the end of the sequences.

Recall $(\mathcal{R})$ and precision $(\mathcal{P})$ indexes are often used for the evaluation of video processing. They are computed as $\mathcal{R}=\frac{C \cap R}{C}$ and $\mathcal{P}=\frac{C \cap R}{R}$, where $C$ is the reference set obtained by expert annotations, $R$ is the set of retrieved frames provided by the recognition module by using the BetP-based criteria, and $C \cap R$ is the number of correctly retrieved frames. In order to assess the method by only one criterion, the $\mathcal{F}_{1}$-measure is used: $\mathcal{F}_{1}=\frac{2 \times \mathcal{R} \times \mathcal{P}}{\mathcal{R}+\mathcal{P}}$ It allows to combine $\mathcal{R}$ and $\mathcal{P}$ (here, the same importance is given to both components). This measure tends to the minimum between $\mathcal{R}$ and $\mathcal{P}$.

Illustration and analysis: Table 1 gathers the $\mathcal{F}_{1}$-measures reflecting action recognition performance for each action of each sequence. An important gain is obtained for all actions by using the Belief Scheduler because (1) it integrates past events while embedding a memory fading and (2) the model change is constrained according to a sequence, both allowing to remove false alarms.

Table 1: $\mathcal{F}_{1}$-measure (in $\%$ ) before and after filtering for actions in pole vault and high jump. The last column is the gain/loss on $\mathcal{F}_{1}$ after both filtering and scheduling.

\begin{tabular}{|c|c|c|c|}
\hline High jump & before & after & gain \\
\hline running & 67.9 & 86.6 & +18.7 \\
jumping & 57.9 & 78.4 & +20.5 \\
falling & 70.1 & 81.0 & +10.9 \\
\hline Sequence & - & 91.2 & - \\
\hline \hline Pole vault & before & after & gain \\
\hline running & 73.6 & 85.6 & +12.0 \\
jumping & 48.6 & 80.9 & +32.3 \\
falling & 64.9 & 78.5 & +13.6 \\
\hline Sequence & - & 88.5 & - \\
\hline \hline
\end{tabular}

The illustration depicted in Figure 9 (for a high jump) shows the efficiency of the approach. Belief on propositions $R_{A}$ ( $A$ is right), $F_{A}$ ( $A$ is false) and $R_{A} \cup F_{A}$ ( $A$ is right or false) are represented.

\section{Conclusion}

The Belief Scheduler of actions proposed for temporal sequence recognition is based on the Transferable Belief Model fusion process.

After the parameters fusion step, the Temporal Belief Filter is used to (i) smooth belief (ii) detect the transition between action using the conflict between model and observation. In order to improve and emphasize the transition between two actions, a cusum of the conflict is computed. Because the human activity can be described by a sequence of actions, we have developed an original Belief Scheduler to improve the state evaluation of each action that composes the concerned activity. The preemption and forcing processing, well known in the System Science community has been adapted in order to re-synchronize the actions belonging to the sequence. The originality of the proposed algorithm is that it is based on the conflict detection and evaluation: conflict between parameters and constraint state models, and conflict between exclusive actions states. An elucidation of the parameters tuning is also provided to facilitate the setting.

The method was successfully applied on 40 real video sequences acquired with a moving camera to recognize athlete jumps such as pole vault and high jump as well as actions in jumps such as running, jumping and falling.

The generalization capability was tested by applying successfully the Belief Scheduler with the same settings on both activities. The future work concern parameters learning and models adaptation based on gradual 
BEFORE
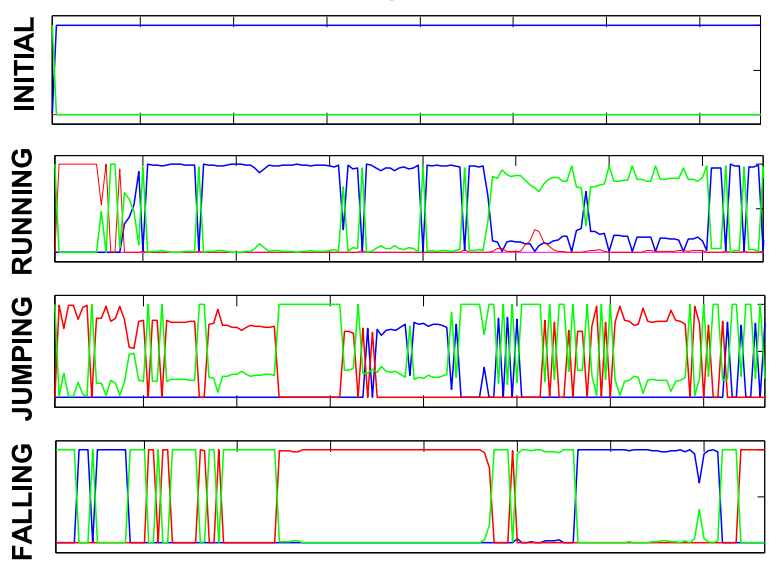

AFTER
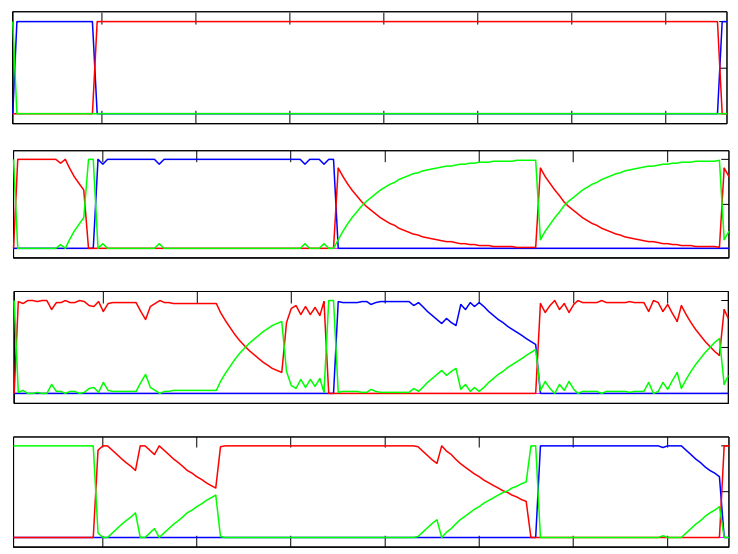

Figure 9: Result of the Belief Scheduler for a high jump sequence. The belief on $R_{A}, F_{A}$ and $R_{A} \cup F_{A}$ are represented respectively in blue, red and green color.

rules. Furthermore, a comparison with ENC [13, 14] will be performed.

\section{Acknowledgement}

This research is partially supported by SIMILAR European excellence network. The authors would like to thank University of Crete for data exchange.

\section{References}

[1] L. Wang, W. Hu, and T. Tan, "Recent developments in human motion analysis," $P R$, vol. 36, no. 3, pp. 585-601, 2003.

[2] M. Lew, N. Sebe, and J. Eakins, "Challenges in image and video retrieval," Lecture notes in Computer Science, ICIVR, vol. 2383, pp. 1-6, 2002.

[3] S. Hongeng, R. Nevatia, and F. Bremond, "Videobased event recognition and probabilistic recognition methods," CVIU, vol. 96, pp. 129-162, 2004.

[4] Y. Luo, T.D. Wu, and J.N. Hwang, "Object-based analysis and interpretation of human motion in sports video sequences by dynamic bayesian networks," CVIU, vol. 92, pp. 196-216, 2003.

[5] E. Ramasso, D. Pellerin, C. Panagiotakis, M. Rombaut, G. Tziritas, and W. Lim, "Spatiotemporal information fusion for human action recognition in videos," in 13th European Signal Processing Conf., Antalya, Turkey, Sept. 2005.

[6] P. Smets and R. Kennes, "The Transferable Belief Model," Artificial Intelligence, vol. 66, no. 2, pp. 191-234, 1994.

[7] E. Ramasso, M. Rombaut, and D. Pellerin, "A temporal belief filter improving human action recognition in videos," in International Conference on Acoustics, Speech and Signal Processing, Toulouse, France, 2006, to appear.
[8] T.G. Robertazzi, "Ten reasons to use divisible load theory," Computer, vol. 36, pp. 63-68, 2003.

[9] C. Panagiotakis and G. Tziritas, "Recognition and tracking of the members of a moving human body," in Articulated Motion and Deformable Objects, 2004, vol. 3179, pp. 86-98.

[10] B. Ristic and P. Smets, "Target identification using belief functions and implication rules," IEEE Trans. Aerospace and Electronic Systems, vol. 41, no. 3, pp. 1097-1102, July 2005.

[11] Z. Elouedi, K. Mellouli, and Ph. Smets, "Assessing sensor reliability for multisensor data fusion within the Transferable Belief Model," IEEE Trans. Systems, Man and Cybernetics, vol. 34, no. 1, pp. 782-787, 2004.

[12] M. Rombaut, I. Jarkass, and T. Denoeux, "State recognition in discrete dynamical systems using petri nets and evidence theory," in ECSQARU, June 1999, pp. 352-361.

[13] H. Xu and P. Smets, "Evidential reasoning with conditionnal belief functions," Uncertainty in Artificial Intelligence, vol. 94, pp. 598-606, 1994.

[14] B. Ben Yaghlane, P. Smets, and K. Mellouli, "Directed evidential networks with conditionnal belief functions," in ECSQARU, 2003, vol. 2711, pp. 291-305.

[15] P. Vannoorenberghe and Ph. Smets, "Partially supervised learning by a Credal EM approach," in ECSQARU, 2005, vol. 3571, pp. 956-967.

[16] J. Reuter and W.F. Tichy, "Logging kernel events on clusters," Future Generation Computer Systems, vol. 22, no. 3, pp. 313-323, 2006.

[17] Ph. Smets, "Decision making in the TBM: the necessity of the pignistic transformation," Int. J. of Approx. Reasoning, vol. 38, pp. 133-147, 2005. 